\title{
Role of Transient Receptor Potential Vanilloid 1 in Electroacupuncture Analgesia on Chronic Inflammatory Pain in Mice
}

\author{
Jun Yang, ${ }^{1}$ Ching-Liang Hsieh, ${ }^{2,3,4,5}$ and Yi-Wen Lin ${ }^{2,5}$ \\ ${ }^{1}$ Department of Acupuncture, China Medical University Hospital, Taichung 40402, Taiwan \\ ${ }^{2}$ College of Chinese Medicine, Graduate Institute of Acupuncture Science, China Medical University, Taichung 40402, Taiwan \\ ${ }^{3}$ College of Chinese Medicine, Graduate Institute of Integrated Medicine, China Medical University, Taichung 40402, Taiwan \\ ${ }^{4}$ Department of Chinese Medicine, China Medical University Hospital, Taichung 40402, Taiwan \\ ${ }^{5}$ Research Center for Chinese Medicine \& Acupuncture, China Medical University, Taichung 40402, Taiwan
}

Correspondence should be addressed to Yi-Wen Lin; yiwenlin@mail.cmu.edu.tw

Received 13 August 2017; Accepted 21 November 2017; Published 12 December 2017

Academic Editor: Jane Hanrahan

Copyright (c) 2017 Jun Yang et al. This is an open access article distributed under the Creative Commons Attribution License, which permits unrestricted use, distribution, and reproduction in any medium, provided the original work is properly cited.

\begin{abstract}
Chronic inflammatory pain may result from peripheral tissue injury or inflammation, increasing the release of protons, histamines, adenosine triphosphate, and several proinflammatory cytokines and chemokines. Transient receptor potential vanilloid 1 (TRPV1) is known to be involved in acute to subacute neuropathic and inflammatory pain; however, its exact mechanisms in chronic inflammatory pain are not elucidated. Our results showed that EA significantly reduced chronic mechanical and thermal hyperalgesia in the chronic inflammatory pain model. Chronic mechanical and thermal hyperalgesia were also abolished in $\mathrm{TRPV1}^{-/-}$mice. TRPV1 increased in the dorsal root ganglion (DRG) and spinal cord (SC) at 3 weeks after CFA injection. The expression levels of downstream molecules such as pPKA, pPI3K, and pPKC increased, as did those of pERK, pp38, and pJNK. Transcription factors ( $\mathrm{pCREB}$ and $\mathrm{pNF} \kappa \mathrm{B}$ ) and nociceptive ion channels (Navl.7 and Navl.8) were involved in this process. Inflammatory mediators such as GFAP, S100B, and RAGE were also involved. The expression levels of these molecules were reduced in EA and TRPV1 ${ }^{-/-}$mice but not in the sham EA group. Our data provided evidence to support the clinical use of EA for treating chronic inflammatory pain.
\end{abstract}

\section{Introduction}

Chronic pain is a pathological condition that persists for more than 3-6 months since the onset of pain in human beings [1]. The chronic pain model produces a secondary mechanical hyperalgesia in the central nervous system (CNS). Epidemiological studies indicate that approximately $9 \%-64 \%$ of people worldwide are afflicted by chronic pain [2]. Chronic inflammatory pain arises when inflammatory mediators are not neutralized, and the overexpression of proinflammatory cytokines and chemokines further leads to peripheral and central sensitization [3]. Chronic inflammatory pain may result from peripheral injury sites, spinal cord, or central brain and is usually difficult to cure. Patients with chronic pain may be occasionally relieved by opioid administration, even though other treatments are ineffective [4]. Chronic pain without curative treatment has increased the mortality rate in patients with heart disease, depression, anxiety, and sleep disturbances $[5,6]$. Patients afflicted by chronic pain always perform reduced exercise and physical activities, and exercise is an intervention to alleviate pain [7]. Chronic inflammatory pain is defined as nociceptive and neuropathic pain caused by inflamed tissue with activated nociceptors and malfunctioning nerves, respectively [8].

Transient receptor potential (TRP) family has six transmembrane domains that can be further categorized into seven subtypes: TRPV (vanilloid), TRPA (ankyrin), TRPC (canonical), TRPM (melastatin), TRPML (mucolipin), TRPP (polycystin), and TRPN (no mechanoreceptor potential C) [9]. TRPV1 channels can respond to heat, touch, pain, and 
physical and chemical stimuli [9]. They can recognize sensory inputs from peripheral nerve terminals including painful signals. The activation of TRPV1 channels subsequently induces cation influx, especially that of $\mathrm{Ca}^{2+}$, and further activates signaling pathways. TRPV1 is well known as a nociceptor in peripheral sensory neurons [10]. TRPV1, also known as a capsaicin receptor, is a nonselective cation channel that can be activated by mechanical, thermal (temperature more than $43^{\circ} \mathrm{C}$ ), and chemical stimuli. The activation of TRPV1 leads to mechanical, thermal, and burning sensations. In some pathological conditions, this may pain. TRPV1 is primarily found in peripheral nociceptive neurons for pain transduction and is also reportedly involved in integrating painful stimuli $[11,12]$.

Acupuncture was first reported to relieve pain in the 1970s by Johnson [13]. Evidence-based studies have suggested that electroacupuncture (EA) can be used to treat learning and memory impairment in rats with cerebral ischemiareperfusion injury [14], epilepsy [15], body weight control [16], and pain [17-20]. In addition, several studies have suggested that acupuncture can significantly increase the release of endogenous opiates [21], serotonin [22], and adenosine [23] to alleviate nociception. The major mechanism underlying acupuncture analgesia is the release of opiates in CNS. The aim of the present study is to examine the role of TRPV1 in chronic inflammatory pain and to test the expression of TRPV1 and related downstream molecules after inducing chronic inflammatory pain in the peripheral dorsal root ganglion (DRG) and central spinal cord (SC).

\section{Methods}

2.1. Animals. Serial experiments were conducted on C57/B6 male mice (ages 8 to 12 weeks) purchased from BioLASCO Co., Ltd, Taipei, Taiwan. Mice were randomly subdivided into 5 groups ( $n=8$ per group): (1) Control, (2) CIP (CFAinduced chronic inflammatory pain), (3) $2 \mathrm{~Hz}$ EA (CFAinduced chronic inflammatory pain with $2 \mathrm{~Hz} E A$ ), (4) sham EA (CFA-induced chronic inflammatory pain with sham EA), and (5) $\mathrm{TRPV1}^{-/-}$(CFA-induced chronic inflammatory pain in TRPV1 ${ }^{-/-}$mice). The sample size required for an alpha of 0.05 and a power of $80 \%$ is 8 animals per group. After arrival, mice were housed in a $12 / 12 \mathrm{~h}$ light/dark cycle with ad libitum water and food. All procedures were approved by the Institute of Animal Care and Use Committee of China Medical University (permit number 2016-061) and conducted in accordance with the Guide for the use of Laboratory Animals by the National Research Council and the ethical guidelines of the International Association for the Study of Pain. The mice suffering was minimized with $1 \%$ isoflurane. The laboratory workers were blinded to treatment allocation during the experiments and analysis.

2.2. Chronic Inflammatory Pain Induction. Mice were anesthetized with $1 \%$ isoflurane and given a single injection of $20 \mu \mathrm{l}$ saline ( $\mathrm{pH} 7.4$, buffered with $20 \mathrm{mM}$ HEPES) or CFA (75\% in saline, $0.5 \mathrm{mg} / \mathrm{ml}$ heat-killed $M$. tuberculosis [Sigma, St. Louis, MO]) in the plantar surface of the hind paw to induce chronic intraplantar inflammation [19]. Behaviour tests are conducted every 3 days after induction of chronic inflammatory pain.

2.3. Electroacupuncture Treatment. Electroacupuncture (EA) was applied using stainless steel needles $\left(0.5^{\prime \prime}\right.$ inch, $32 \mathrm{G}$, Yu-Kuang, Taiwan) inserted into the muscle layer to a depth of $2-3 \mathrm{~mm}$ at the bilateral ST36 acupuncture point. The ST36 acupuncture point is located on the tibialis anterior muscle, approximately $1 / 6$ of the distance from the patella to the lateral malleolus. Disposable needles with a diameter of $0.30 \mathrm{~mm}$ and a length of $13 \mathrm{~mm}$ (Yu-Kuang Acupuncture Instrument Co., Taiwan) were inserted into the muscle layer at both ST36 acupuncture points to a depth of 2-3 mm. EA was administered at days 15,18 , and 21 after the CFA injection. A stimulator (Trio 300, Ito, Japan) delivered 100- $\mu$ s square pulses of $1 \mathrm{~mA}$ for $15 \mathrm{~min}$ at $2 \mathrm{~Hz}$. EA was applied at nonacupoint to be set as sham control group (upper gluteal muscle).

2.4. Animal Behaviour of Mechanical and Thermal Hyperalgesia. Mechanical and thermal sensitivities were tested at 3 days after intraplantar injection of CFA. All experiments were performed at room temperature (approximately $25^{\circ} \mathrm{C}$ ) and the stimuli were applied only when the animals were calm but not sleeping or grooming. Mechanical sensitivity was measured by testing the force of responses to stimulation with 3 applications of electronic von Frey filaments (North Coast Medical, Gilroy, CA, USA). At $1 \mathrm{~h}$ after mechanical testing, thermal pain was measured with 3 applications using Hargraves' test IITC analgesiometer (IITC Life Sciences, Woodland Hills, CA, USA) [19].

2.5. Immunoblotting Assay. After animal behaviour test (at day 21), mice DRG and SC dorsal horn were immediately excised to extract proteins. Total proteins were prepared by homogenized tissue in lysis buffer containing $50 \mathrm{mM}$ Tris$\mathrm{HCl}$ pH 7.4, 250 mM NaCl, 1\% NP-40, 5 mM EDTA, 50 mM $\mathrm{NaF}, 1 \mathrm{mM}$ Na3VO4, $0.02 \% \mathrm{NaN} 3$, and 1x protease inhibitor cocktail (AMRESCO). The extracted proteins (30 $\mu \mathrm{g}$ per sample assessed by BCA protein assay) were subjected to $8 \%$ SDSTris glycine gel electrophoresis and transferred to a PVDF membrane. The membrane was blocked with 5\% nonfat milk in TBS-T buffer $(10 \mathrm{mM}$ Tris $\mathrm{pH} 7.5,100 \mathrm{mM} \mathrm{NaCl}, 0.1 \%$ Tween 20), incubated with primary antibody against TRPV1 ( 95 kDa, 1:1000, Alomone, Israel), pPKA ( 51 kDa, 1:1000, Millipore, USA), pPI3K ( 125 kDa, 1: 1000, Millipore, USA), pPKC ( 80-82 kDa, 1:1000, Millipore, USA), pERK1/2 ( 42-44 kDa, 1:1000, Millipore, USA), pp38 ( 41 kDa, 1:1000, Millipore, USA), pJNK ( 42 kDa, 1:1000, Millipore, USA), pAkt ( $\sim 60 \mathrm{kDa}, 1: 1000$, Millipore, USA), pmTOR ( 60 kDa, 1:500, Millipore, USA), pCREB ( 43 kDa, 1:1000, Millipore, USA), pNF $\kappa$ B ( $65 \mathrm{kDa}, 1: 1000$, Millipore, USA), Nav1.7 ( $230 \mathrm{kDa}, 1: 1000$, Millipore, USA), Nav1.8 ( 220 kDa, 1:1000, Millipore, USA), GFAP ( $50 \mathrm{kDa}, 1: 1000$, Millipore, USA), S100B ( 10-15 kDa, 1:1000, Millipore, USA), and RAGE antibody ( $48 \mathrm{kDa}, 1: 1000$, Millipore, USA), in TBS-T with $1 \%$ bovine serum albumin, and incubated for 1 hour at room temperature. Peroxidase-conjugated anti-rabbit antibody $(1: 5000)$ was used as a secondary antibody. The bands were visualized by an enhanced chemiluminescence substrate 


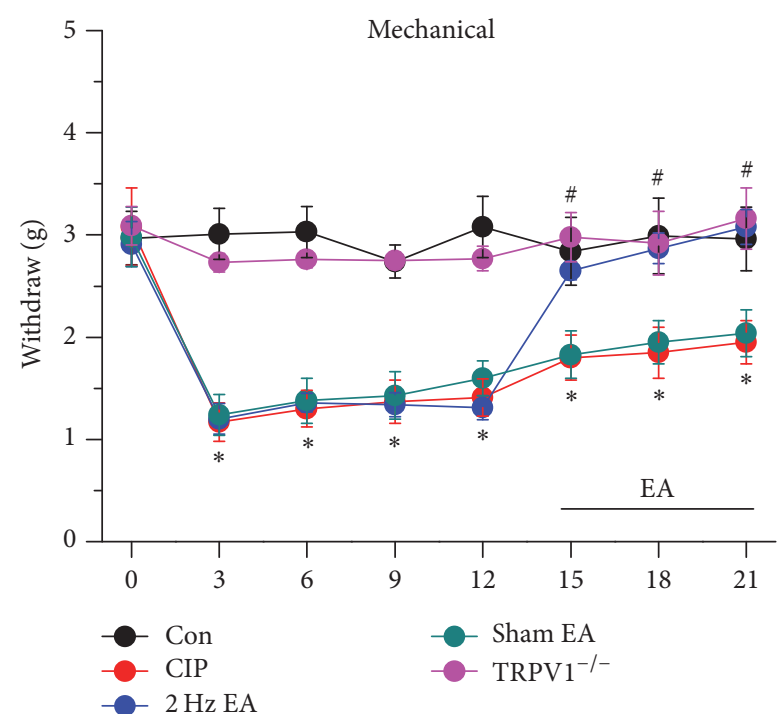

(a)

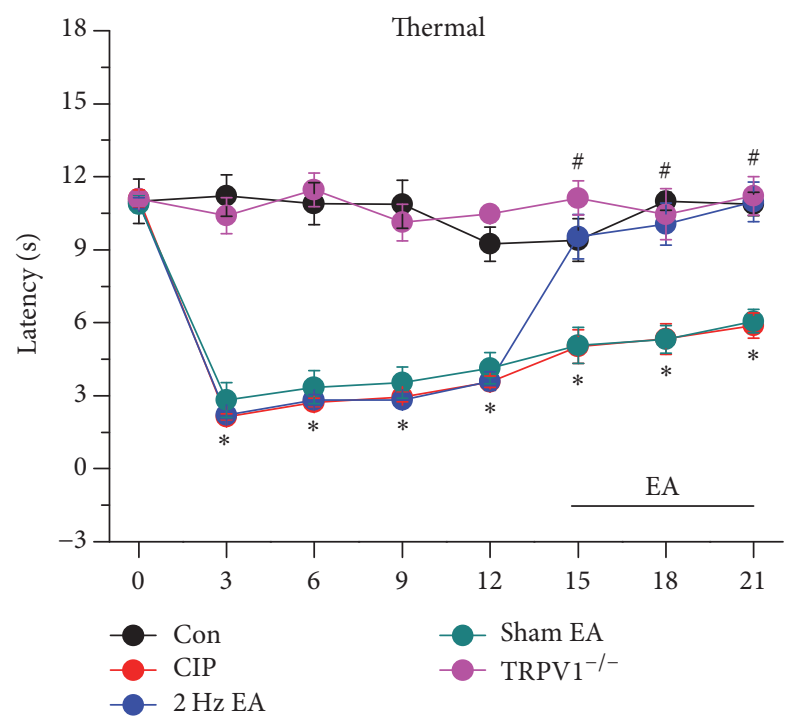

(b)

FIGURE 1: Mechanical and thermal withdrawal thresholds in each group of mice. Normal saline injection (Con group, $n=8$ ), CIP (CFAinduced chronic inflammatory pain), $2 \mathrm{~Hz}$ EA (CFA-induced chronic inflammatory pain with $2 \mathrm{~Hz}$ EA), sham EA (CFA-induced chronic inflammatory pain with sham EA), and $\mathrm{TRPV1}^{-/-}$(CFA-induced chronic inflammatory pain in $\mathrm{TRPV1}^{-/-}$mice). ${ }^{*} p<0.05$ versus Con. ${ }^{\#} p<0.05$ versus CIP group.

kit (PIERCE) with LAS-3000 Fujifilm (Fuji Photo Film Co. Ltd). Where applicable, the image intensities of specific bands were quantified with NIH ImageJ software (Bethesda, MD, USA) [19].

2.6. Statistical Analysis. All statistic data are presented as the mean \pm standard error. Statistical significance between control, CIP, $2 \mathrm{~Hz}$ EA, sham EA, and TRPV1 ${ }^{-/-}$groups was tested using the ANOVA test, followed by a post hoc Tukey's test $(p<0.05$ was considered statistically significant).

\section{Results}

Acupuncture has been used for thousands of years to manage pain, albeit with unclear mechanisms. We used mechanical and thermal pain behaviour to examine the role of TRPV1 in EA analgesia on CFA-induced chronic inflammatory pain model. Mechanical hyperalgesia was not induced at day 21 in control mice injected with normal saline (Figure 1(a), mechanical force $=2.96 \pm 0.31 \mathrm{~g}, n=8$ ). Chronic mechanical hyperalgesia was induced after CFA injection on day 21 (Figure 1(a), $1.95 \pm 0.21 \mathrm{~g}, n=8$ ). We then identified that $2 \mathrm{~Hz}$ EA significantly attenuated CFA-induced chronic mechanical hyperalgesia on day 21 (Figure $1(\mathrm{a}), 3.08 \pm 0.17 \mathrm{~g}$, $n=8)$. With acupoint specificity, sham EA did not alter mechanical hyperalgesia (Figure 1(a), $2.04 \pm 0.23 \mathrm{~g}, n=8$ ). Our results further suggested that TRPV1 was crucial for chronic pain because chronic mechanical hyperalgesia was not induced in $\mathrm{TRPV}^{-/-}$mice (Figure 1(a), $3.16 \pm 0.3 \mathrm{~g}$, $n=8$ ). We further investigated if thermal hyperalgesia was similarly affected. Injection of normal saline did not induce thermal hyperalgesia (Figure 1(b), thermal latency $=10.87 \pm$ $0.48 \mathrm{~g}, n=8$ ). Injection of CFA reliably induced chronic thermal hyperalgesia (Figure 1(b), $5.89 \pm 0.52 \mathrm{~g}, n=8$ ) and it was further reduced by $2 \mathrm{~Hz}$ EA stimulation (Figure $1(\mathrm{~b})$, $10.96 \pm 0.81 \mathrm{~g}, n=8$ ). The effect was not observed in the sham-operated group (Figure 1(b), $6.06 \pm 0.48 \mathrm{~g}, n=8$ ). Moreover, thermal hyperalgesia induced by CFA was not seen in TRPV1 ${ }^{-1-}$ mice (Figure 1(b), $11.22 \pm 0.79 \mathrm{~g}, n=8$ ). The aforementioned results indicate that EA dramatically decreased both chronic mechanical and thermal hyperalgesia in a mouse chronic inflammatory pain model.

Subsequently, we used western blot analysis to determine if chronic inflammatory pain could change the protein level of TRPV1 and the associated downstream molecules. Our data suggested that TRPV1 was expressed in DRG in control mice (Figure 2(a), $100.1 \pm 7.31 \%, n=8$ ) and then increased in chronic inflammatory pain in mice (Figure 2(a), $168.89 \pm$ $11.6 \%, n=8)$. In addition, $2 \mathrm{~Hz}$ EA significantly reduced the overexpression of TRPV1 (Figure 2(a), $131.23 \pm 11.48 \%$, $n=8)$. Sham EA treatment did not alter this observation (Figure 2(a), $156.69 \pm 10.88 \%, n=8$ ).

We then addressed the effects of EA on TRPV1 and the associated downstream protein kinases. We showed that pPKA, pPI3K, and pPKC levels increased in chronic inflammatory pain in mice DRG (Figure 2(a), $128.3 \pm 8.86 \%$, $132.13 \pm 11.59 \%$, and $137.38 \pm 13.93 \%, n=8)$ and then reversed by EA (Figure 2(a), $93.51 \pm 5.59 \%$, $95.28 \pm 6.33 \%$, and $105.94 \pm 11.89 \%, n=8$ ) but not by the sham treatment (Figure 2(a), $128.55 \pm 10.41 \%, 126.95 \pm 10.45 \%$, and $132.02 \pm$ $12.15 \%, n=8)$. The overexpression of such protein kinases decreased in $\mathrm{TRPV}^{-/-}$mice (Figure 2(a), $90.21 \pm 6.36 \%$, $92.98 \pm 7.89 \%$, and $104.57 \pm 10.45 \%, n=8)$. We then examined the downstream protein kinases in all groups. We showed that pERK, pp38, pJNK, and pAkt signaling pathways 


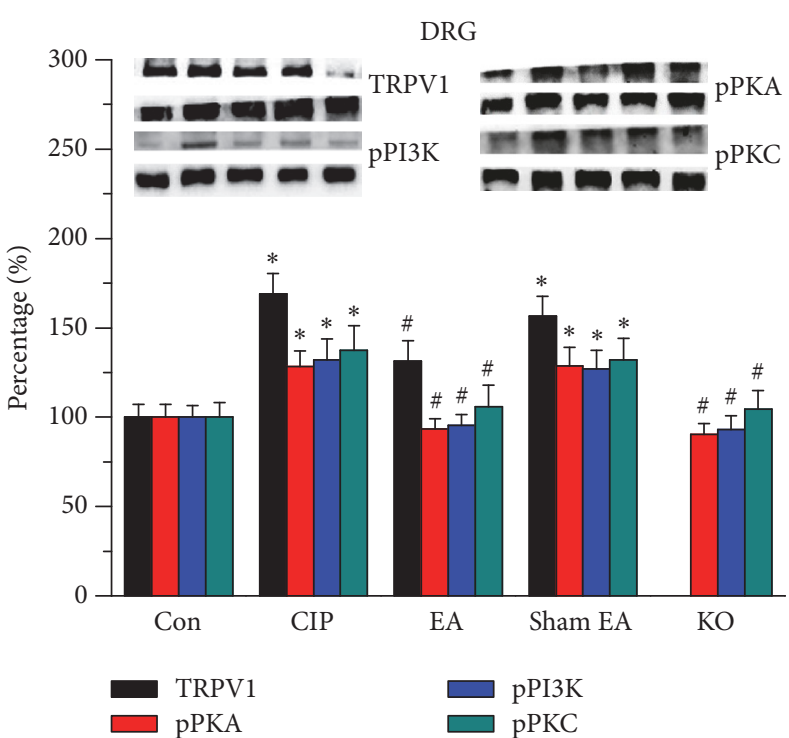

(a)

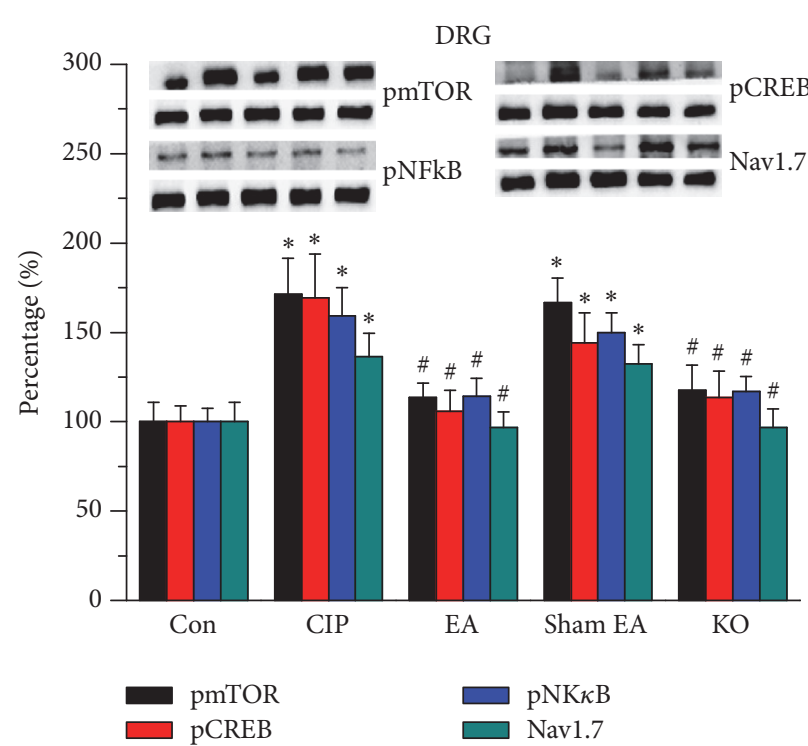

(c)

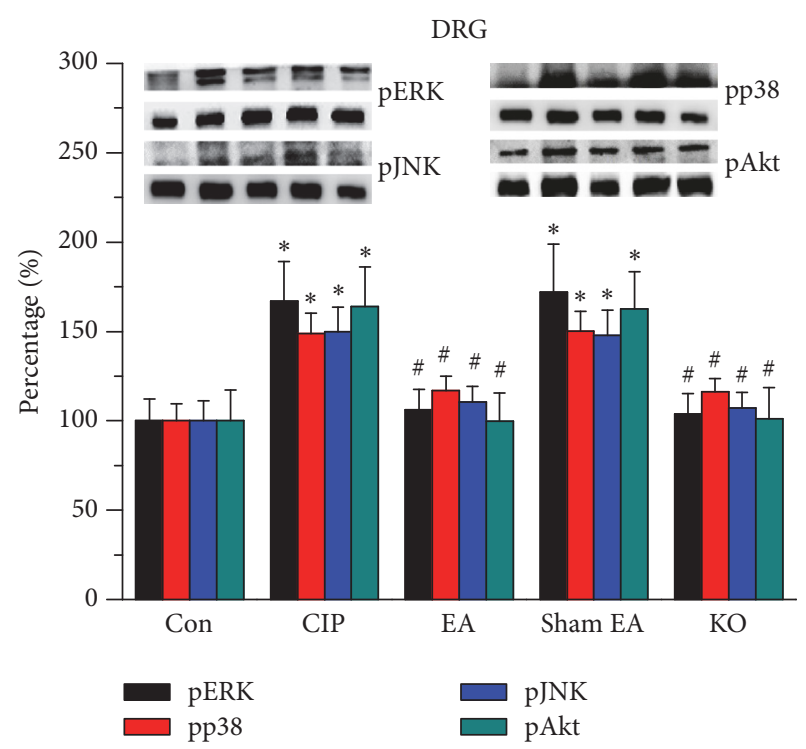

(b)

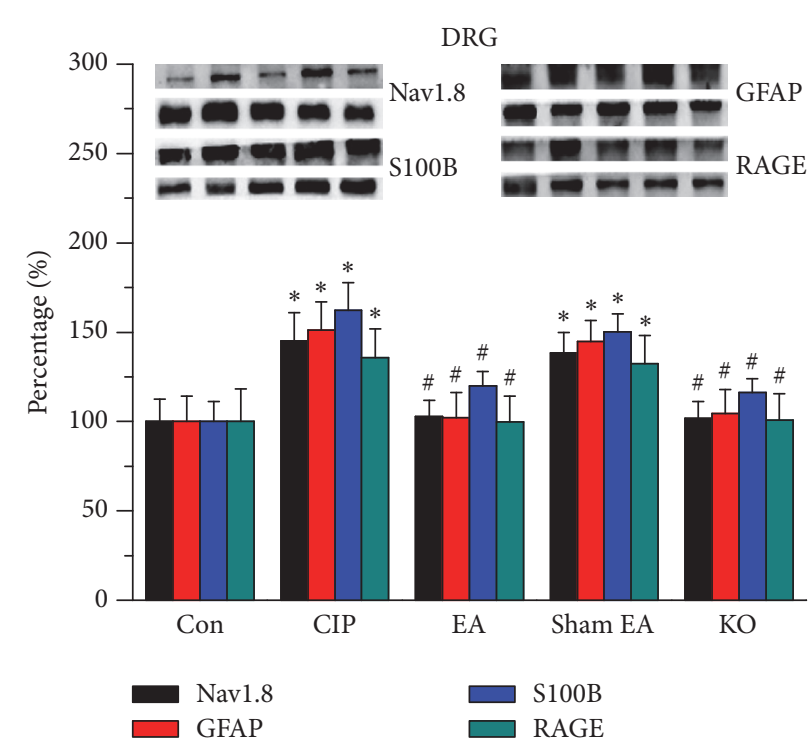

(d)

FIGURE 2: Expression levels of TRPV1-associated signaling pathway in mice DRG. (a) TRPV1, pPKA, pPI3K, and pPKC. (b) pERK, pp38, pJNK, and pAkt. (c) pmTOR, pCREB, pNF $\kappa$ B, and Navl.7. (d) Nav1.8, GFAP, S100B, and RAGE expression levels in tissues from Con, CIP, $2 \mathrm{~Hz}$ EA, sham EA, and TRPV1 ${ }^{-/-}$groups (from left to right). Con: control; CIP: chronic inflammatory pain; EA: electroacupuncture; sham EA: sham electroacupuncture; KO: TRPV1 knockout mice. ${ }^{*} p<0.05$ versus Con. ${ }^{\#} p<0.05$ versus CUP group. The western blot bands at the top show the target protein. The lower bands are internal controls ( $\beta$-actin or $\alpha$-tubulin).

were upregulated in chronic inflammatory pain in mice (Figure 2(b), 167.08 $\pm 22.08 \%, 148.74 \pm 11.5 \%, 149.67 \pm 13.82 \%$, and $163.93 \pm 22.15 \%, n=8)$. The overexpression of these molecules was further reduced by EA (Figure 2(b), $106.15 \pm$ $11.31 \%, 117.05 \pm 7.77 \%, 110.69 \pm 8.66 \%$, and $99.77 \pm 15.66 \%$, $n=8$ ) but not by the sham treatment (Figure 2(b), $171.91 \pm$ $26.85 \%, 150.14 \pm 11.28 \%, 147.82 \pm 14.19 \%$, and $162.47 \pm$ $21.12 \%, n=8)$. All signals were decreased to control level in $\mathrm{TRPV1}^{-/-}$mice (Figure 2(b), 103.74 $\pm 11.65 \%, 116.09 \pm 7.58 \%$, $107.08 \pm 8.87 \%$, and $101.15 \pm 17.55 \%, n=8)$. Similar results were also observed in pmTOR expression (Figure 2(c), $n=8$ ).
We further illustrated that the transcription factors pCREB and $\mathrm{pNK} \kappa \mathrm{B}$ also increased in chronic inflammatory pain in mice (Figure 2(c), $169.32 \pm 24.39 \%$, and $159.16 \pm$ $16.03 \%, n=8)$, which was then attenuated by EA (Figure 2(c), $105.93 \pm 11.64 \%$ and $113.50 \pm 8.19 \%, n=8$ ) but not by the sham treatment (Figure 2(c), $136.36 \pm 12.99 \%$ and $143.96 \pm 17.4 \%, n=8)$. The overexpression of pCREB and $\mathrm{pNK} \kappa \mathrm{B}$ was not observed in $\mathrm{TRPV1} 1^{-/-}$mice (Figure 2(c), $13.67 \pm 14.7 \%$, and $117.48 \pm 14.24 \%, n=8$ ). Furthermore, the expression levels of the nociceptive channels Nav1.7 and Nav1.8 increased in chronic pain in mice (Figures 2(c) and 


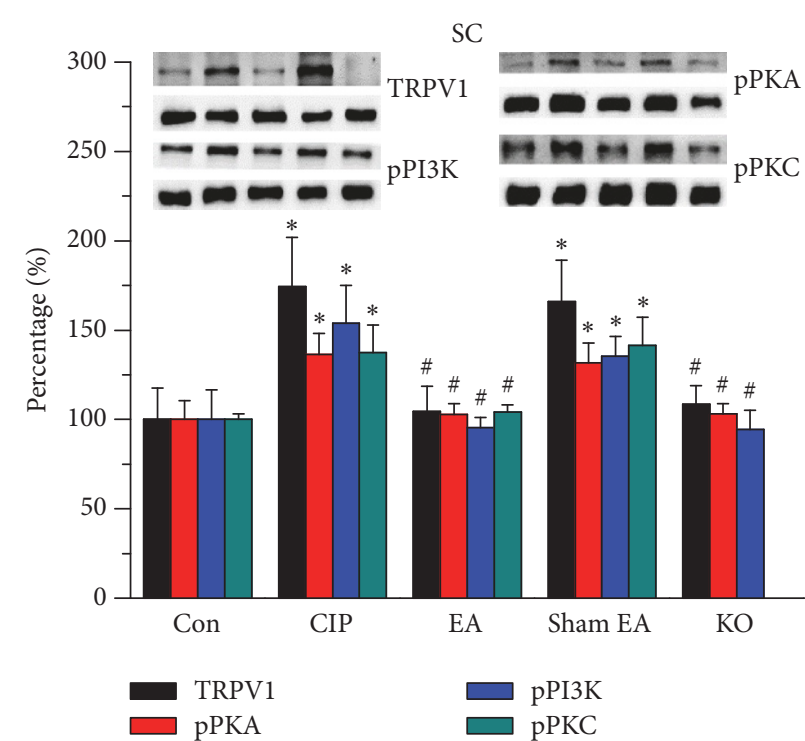

(a)

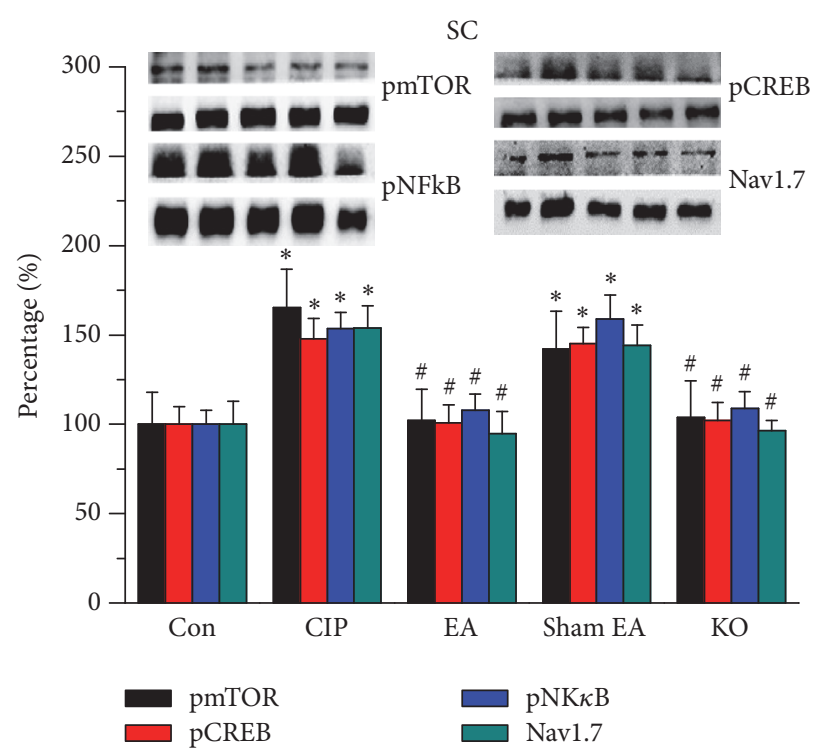

(c)

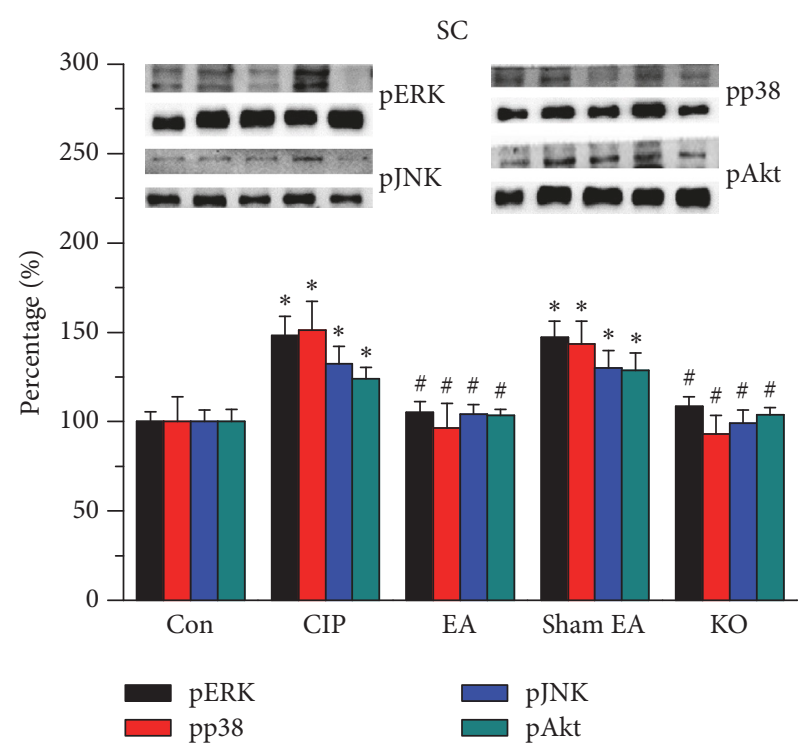

(b)

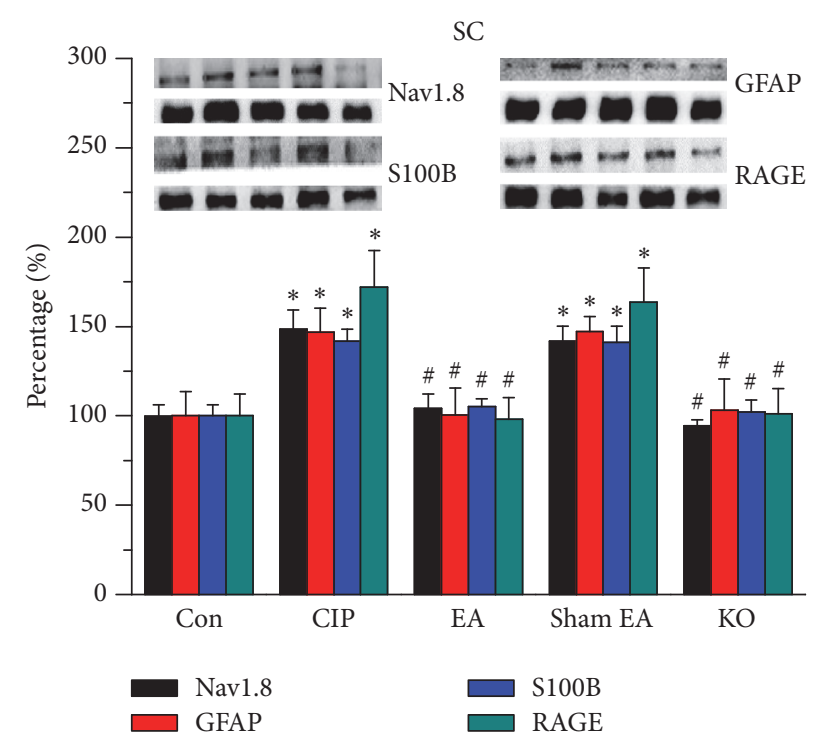

(d)

FIGURE 3: Expression levels of TRPV1-associated signaling pathway in mice SC. (a) TRPV1, pPKA, pPI3 K, and pPKC. (b) pERK, pp38, pJNK, and pAkt. (c) pmTOR, pCREB, pNF $\kappa$ B, and Nav1.7. (d) Nav1.8, GFAP, S100B, and RAGE expression levels in tissues from Con, CIP, 2 Hz EA, sham EA, and TRPV1 ${ }^{-1-}$ groups (from left to right). Con: control; CIP: chronic inflammatory pain; EA: electroacupuncture; sham EA: sham electroacupuncture; KO: TRPV1 knockout mice. ${ }^{*} p<0.05$ versus Con. ${ }^{\#} p<0.05$ versus CUP group. The western blot bands at the top show the target protein. The lower bands are internal controls ( $\beta$-actin or $\alpha$-tubulin).

2(d), $135.86 \pm 12.99 \%$ and $139.16 \pm 16.03 \%, n=8$ ), which were reduced by EA and TRPV1 deletions but not in the sham treated group (Figures 2(c) and 2(d), $n=8$ ). Figure 2(d) shows that the levels of GFAP (satellite cell marker in DRG), S100B, and RAGE were upregulated in chronic inflammatory pain in mice $(145.18 \pm 15.85,151.34 \pm 15.78 \%$, and $136.26 \pm 13.29 \%)$. EA $(102.96 \pm 8.83 \%, 119.87 \pm 8.22 \%$, and $102.08 \pm 14.19 \%)$ and TRPV1 deletion (104.39 $\pm 13.37 \%$, $116.14 \pm 7.95 \%$, and $101.69 \pm 9.42 \%)$ significantly reduced inflammatory biomarkers but not in the sham treated group $(144.5 \pm 11.7 \%, 150.21 \pm 9.9 \%$, and $132.2 \pm 16.06 \%)$.
To determine whether EA alters TRPV1 and associated molecules and regulate pain, we tested aforementioned pathways in SC tissue of the mice. We indicated that TRPV1 was expressed in the normal SC (Figure 3(a), $100.0 \% \pm 3.17 \%$, $n=8$ ), and that the expression level increased in chronic inflammatory pain in mice SC (Figure 3(a), $137.55 \% \pm 15.46 \%$, $n=8)$. In particular, EA significantly reduced TRPV1 increase in SC (Figure 3(a), 104.23\% $\pm 3.84 \%, n=8$ ), and a similar result was observed in TRPV1 ${ }^{-/-}$mice (Figure 3(a), $n=8$ ) but not in sham-operated group (Figure 3(a), $141.46 \% \pm 15.92 \%, n=8)$. Then, we tested the expression 
levels of pPKA, pPI3K, and pPKC. We showed that pPKA, $\mathrm{pPI} 3 \mathrm{~K}$, and $\mathrm{pPKC}$ were all increased in chronic inflammatory pain in mice SC (Figure 3(a), $153.78 \pm 21.35 \%, 136.56 \pm$ $11.51 \%$, and $174.29 \pm 17.34 \%, n=8)$. This phenomenon is reversed by EA (Figure 3(a), $95.35 \pm 5.83 \%, 102.73 \pm 6.16 \%$, and $104.31 \pm 14.35 \%, n=8)$ and in TRPV1 knockout mice (Figure 3(a), $94.34 \pm 10.82 \%, 103.24 \pm 5.68 \%$, and $108.44 \pm$ $10.59 \%, n=8)$. A similar pattern was not found in the sham EA group (Figure 3(a), $135.24 \pm 11.27 \%, 131.68 \pm 10.98 \%$, and $166.03 \pm 23.24 \%, n=8$ ). We further showed that pERK, pp38, pJNK, and pAkt expression levels increased in SC of CFA-induced chronic inflammatory pain in mice (Figure 3(b), $132.4 \pm 9.81,123.85 \pm 6.57 \%, 148.3 \pm 10.76 \%$, and $151.04 \pm 16.12 \%, n=8)$, which were further attenuated by EA (Figure 3(b), \%, 104.3 $\pm 5.12,103.42 \pm 3.52 \%, 105.32 \pm$ $5.82 \%$, and $96.52 \pm 13.83 \%, n=8)$ and TRPV1 gene deletion treatment (Figure 3(b), 99.17 $\pm 7.18 \%, 103.65 \pm 4.09 \%, 108.66 \pm$ $5.16 \%$, and $93.11 \pm 10.49 \%, n=8)$ but not in the sham group (Figure 3(b), $130.01 \pm 9.84 \%, 128.64 \pm 9.73 \%, 147.09 \pm 9.02 \%$, and $143.51 \pm 12.81 \%, n=8)$. Similar results were also observed in pmTOR expression (Figure 2(c), $n=8$ ).

The expression levels of $\mathrm{pCREB}$ and $\mathrm{pNK} \kappa \mathrm{B}$ increased in CFA-induced chronic inflammatory pain in mice (Figure 3(c), $165.26 \% \pm 21.54 \%$ and $153.85 \% \pm 12.51 \%, n=8$ ), which was reduced by EA (Figure 3(c), $102.2 \% \pm 17.53 \%$ and $94.58 \% \pm 12.46 \%, n=8$ ) and TRPV1 null mice (Figure 3(c), $103.86 \% \pm 20.41 \%$ and $96.53 \% \pm 5.44 \%, n=8$ ) but not in the sham-operated group (Figure 3(c), 159.02\% $\pm 13.4 \%$ and $142.18 \% \pm 20.9 \%, n=8)$. The expression levels of Nav1.7 and Nav1.8 showed similar results (Figure 3(c), $n=8$ ). Similar results were also observed for inflammatory factors such as GFAP, S100B, and RAGE (Figure 3(d), $n=8$ ). The aforementioned results provide evidence that the TRPV1 signaling pathway is important in the central nerve system of mice with chronic inflammatory pain.

\section{Discussion}

TRPV1 is known to be involved in the perception of inflammatory and thermal pain, especially pain from heat, which exceeds $43^{\circ} \mathrm{C}$ [10]. The activation of TRPV1 can further initiate $\mathrm{Ca}^{2+}$ influx for neuronal depolarization $[24,25]$. The downregulation of TRPV1 function results in insensitivity to high temperature stimulation, radial heat, and hot-plate tests [26]. Inflammatory mediators can induce hyperalgesia to activate TRPV1, suggesting their important roles in inflammatory pain [27]. Injection of TRPV1 antagonist capsazepine reduced thermal hyperalgesia in the inflammatory pain model $[28,29]$. In inflammatory pain, proinflammatory mediators induce neuronal hyperactivity, initiating TRPV1 activation [27]. In the present study, we suggested that EA can reduce the overexpression of GFAP, S100B, and RAGE to reduce chronic inflammatory pain. The release of NGF can further bind the TrkA receptor and activate the PI3 kinase and Src kinase to phosphorylate TRPV1 [30]. TRPV1 is also regulated by phosphatidylinositol 4,5-bisphosphate, increasing the phosphorylation of TRPV1 by protein kinases [31-34]. Protease-activated receptor 2 is suggested to sensitize the TRPV1 receptors for thermal hyperalgesia by activating protein kinase A or protein kinase Ce signals [35]. Recent study, using a rat model of monoarthritis, showed that IL1, IL-6, and TNF- $\alpha$ were activated after CFA injection [36]. Another study also indicated the relationship of inflammatory pain with TRPV1 and associated molecules in mice DRG and SC [17]. Lu et al. reported that the expression of TRPV1 and associated signaling pathways was increased after the CFA injection; the overexpression can be further reduced by EA treatment and TRPV1 gene deletion, suggesting that TRPV1 knockout mice are resistant to inflammatory pain [19]. Our data showed that these protein kinases increased in chronic pain model and can be further reduced by EA and TRPV1 gene deletions. The aforementioned mechanisms indicated that TRPV1 was involved in chronic inflammatory pain models and served as key receptors to sense mechanical and thermal pain.

Wu et al. indicated an abundance of TRPV1 in anatomical layers of ST36, especially in muscle layer. Immunofluorescence data also showed that TRPV1 is expressed in both neural and nonneural cells at ST36 acupoint. Injection of capsaicin, a TRPV1 agonist, into ST36 acupoints can relieve inflammatory pain as effectively as manual acupuncture treatment, replicating the analgesic effect of acupuncture [37]. Zhang et al. indicated that EA at ST36 acupoints could attenuate cancer-induced pain through attenuating TRPV1 expression in both mRNA and protein levels in DRG from tumor-bearing rats. They found that the injection of cancer cells could alleviate the paw withdrawal threshold and increase thermal hyperalgesia. The phenomenon could be further reversed by EA at the ST36 acupoints at $2 \mathrm{~Hz}$ low frequency [38]. Chen et al. documented that EA at bilateral ST36 acupoints can reduce mechanical and thermal hyperalgesia induced by injection of CFA or carrageenan into the plantar. They indicated that TRPV1 and TRPV4 increased in inflammatory pain and can be further attenuated by EA stimulation at the peripheral DRG neurons. These results not only indicated pain relief by EA but also suggested that EA could reduce the overexpression of TRPV1 and TRPV4 in small-to-large diameter neurons [39]. Paradoxically, patients receiving chronic opiates reportedly experience hyperalgesia. The hyperalgesia was accompanied with increased TTX$\mathrm{R}$ sodium and TRPV1 channels in sensory neurons [40]. In the present study, we suggested that EA is beneficial for chronic inflammatory pain management by controlling TRPV1 overexpression.

In the present study, our data suggested that EA can significantly reduce chronic inflammatory pain by downregulating the increased signaling of TRPV1 pathway from peripheral DRG to central SC. First, $2 \mathrm{~Hz}$ EA reduced CFA-induced chronic mechanical and thermal hyperalgesia. Second, the TRPV1 signaling pathway was increased in chronic inflammatory pain in mice DRG and further reversed by EA but not in the sham-operated group. Furthermore, similar results were also obtained by deleting the TRPV1 gene. Moreover, we showed that TRPV1 and downstream molecules were also altered in SC supporting the role for central sensitization. Taken together, these mechanisms provide a clear signal of TRPV1 and relevant molecules (Figure 4). 


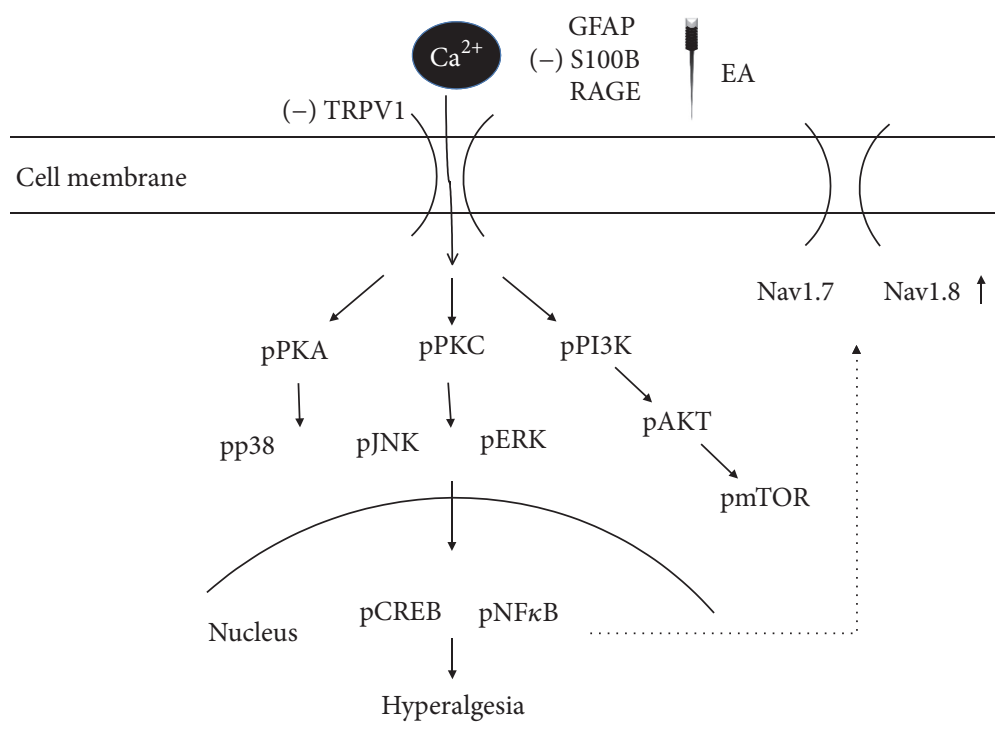

FIGURE 4: Schematic illustration of possible mechanisms of TRPV1 in CFA-induced chronic inflammatory pain in mice model. We showed that TRPV1 is an important receptor in mice chronic inflammatory pain model. The activation of TRPV1 increases the expression of pPKA, pPI3K, pPKC, pAkt, and pmTOR. Furthermore, pERK, pp38, pJNK, pNF $\kappa$ B, and pCREB are also increased. Moreover, nociceptive Nav1.7 and Nav1.8 are increased for pain conduction in both peripheral DRG and central SC. Inflammatory factors such as GFAP, S100B, and RAGE are also involved in this process.

These findings provide significant evidence to practice the clinical application of EA to treat chronic inflammatory pain.

\section{Conflicts of Interest}

The authors declare no conflicts of interest.

\section{Acknowledgments}

This work was supported by CMU under the Aim for Top University Plan of the Ministry of Education, Taiwan, MOST 104-2320-B-039-010, CMU105-S-09.

\section{References}

[1] H. I. Andersson, G. Ejlertsson, I. Leden, and C. Rosenberg, "Chronic pain in a geographically defined general population: Studies of differences in age, gender, social class, and pain localization," The Clinical Journal of Pain, vol. 9, no. 3, pp. 174$182,1993$.

[2] O. A. Steingrimsdottir, T. Landmark, G. J. Macfarlane, and C. S. Nielsen, "Defining chronic pain in epidemiological studies - a systematic review and meta-analysis," Pain, vol. 158, no. 11, pp. 2092-2107, 2017.

[3] I. M. Chiu, C. A. von Hehn, and C. J. Woolf, "Neurogenic inflammation and the peripheral nervous system in host defense and immunopathology," Nature Neuroscience, vol. 15, no. 8, pp. 1063-1067, 2012.

[4] R. Chou, J. A. Turner, E. B. Devine et al., "The effectiveness and risks of long-term opioid therapy for chronic pain: a systematic review for a national institutes of health pathways to prevention workshop," Annals of Internal Medicine, vol. 162, no. 4, pp. 276286, 2015.
[5] M. Fitzgibbon, D. P. Finn, and M. Roche, "High Times for Painful Blues: The Endocannabinoid System in PainDepression Comorbidity," The International Journal of Neuropsychopharmacology, vol. 19, no. 3, p. pyv095, 2015.

[6] B. T. Baune, R. N. Caniato, M. A. Garcia-Alcaraz, and K. Berger, "Combined effects of major depression, pain and somatic disorders on general functioning in the general adult population," Pain, vol. 138, no. 2, pp. 310-317, 2008.

[7] L. J. Geneen, R. A. Moore, C. Clarke, D. Martin, L. A. Colvin, and B. H. Smith, "Physical activity and exercise for chronic pain in adults: an overview of Cochrane Reviews," Cochrane Database of Systematic Reviews, vol. 4, Article ID CD011279, 2017.

[8] C. Wang and C. Wang, "Anti-nociceptive and anti-inflammatory actions of sulforaphane in chronic constriction injuryinduced neuropathic pain mice," Inflammopharmacology, vol. 25, no. 1, pp. 99-106, 2017.

[9] A. P. Christensen and D. P. Corey, "TRP channels in mechanosensation: Direct or indirect activation?" Nature Reviews Neuroscience, vol. 8, no. 7, pp. 510-521, 2007.

[10] S. R. Eid and D. N. Cortright, "Transient receptor potential channels on sensory nerves," Handbook of Experimental Pharmacology, vol. 194, pp. 261-281, 2009.

[11] S. M. Huang, T. Bisogno, M. Trevisani et al., "An endogenous capsaicin-like substance with high potency at recombinant and native vanilloid VR1 receptors," Proceedings of the National Acadamy of Sciences of the United States of America, vol. 99, no. 12 , pp. 8400-8405, 2002.

[12] M. Cui, P. Honore, C. Zhong et al., "TRPV1 receptors in the CNS play a key role in broad-spectrum analgesia of TRPV1 antagonists," The Journal of Neuroscience, vol. 26, no. 37, pp. 9385-9393, 2006.

[13] E. Johnson, "CDA assists in oral surgery performed under acupuncture anesthesia," The Dental Assistant, vol. 42, no. 5, p. $16,1973$. 
[14] C.-T. Kuo, Y.-W. Lin, N.-Y. Tang, C.-Y. Cheng, and C.-L. Hsieh, "Electric stimulation of the ears ameliorated learning and memory impairment in rats with cerebral ischemia-reperfusion injury," Scientific Reports, vol. 6, Article ID 20381, 2016.

[15] Y. W. Lin and C. L. Hsieh, "Auricular electroacupuncture reduced inflammation-related epilepsy accompanied by altered TRPA1, $\mathrm{pPKC} \alpha, \mathrm{pPKC} \varepsilon$, and $\mathrm{pERk} 1 / 2$ signaling pathways in kainic acid-treated rats," Mediators of Inflammation, vol. 2014, Article ID 493480, 9 pages, 2014.

[16] M. Choowanthanapakorn, K.-W. Lu, J. Yang, C.-L. Hsieh, and Y.-W. Lin, "Targeting TRPV1 for Body Weight Control using TRPV1-/-Mice and Electroacupuncture," Scientific Reports, vol. 5, Article ID 17366, 2015.

[17] H.-Y. Liao, C.-L. Hsieh, C.-P. Huang, and Y.-W. Lin, "Electroacupuncture Attenuates CFA-induced Inflammatory Pain by suppressing Nav1.8 through S100B, TRPV1, Opioid, and Adenosine Pathways in Mice," Scientific Reports, vol. 7, Article ID 42531, 2017.

[18] K.-W. Lu, C.-L. Hsieh, J. Yang, and Y.-W. Lin, "Effects of electroacupuncture in a mouse model of fibromyalgia: role of N-methyl-D-aspartate receptors and related mechanisms," Acupuncture in Medicine, vol. 35, no. 1, pp. 59-68, 2017.

[19] K.-W. Lu, C.-K. Hsu, C.-L. Hsieh, J. Yang, and Y.-W. Lin, "Probing the effects and mechanisms of electroacupuncture at ipsilateral or contralateral ST36-ST37 acupoints on CFAinduced inflammatory pain," Scientific Reports, vol. 6, Article ID 22123, 2016.

[20] L. Yen, C. Hsieh, H. Hsu, and Y. Lin, “Targeting ASIC3 for Relieving Mice Fibromyalgia Pain: Roles of Electroacupuncture, Opioid, and Adenosine," Scientific Reports, vol. 7, p. 46663, 2017.

[21] J. S. Han, "Acupuncture: neuropeptide release produced by electrical stimulation of different frequencies," Trends in $\mathrm{Neu}$ rosciences, vol. 26, no. 1, pp. 17-22, 2003.

[22] F.-C. Chang, H.-Y. Tsai, M.-C. Yu, P.-L. Yi, and J.-G. Lin, “The central serotonergic system mediates the analgesic effect of electroacupuncture on Zusanli (ST36) acupoints," Journal of Biomedical Science, vol. 11, no. 2, pp. 179-185, 2004.

[23] N. Goldman, M. Chen, T. Fujita et al., "Adenosine A1 receptors mediate local anti-nociceptive effects of acupuncture," Nature Neuroscience, vol. 13, no. 7, pp. 883-888, 2010.

[24] A. J. Fenwick, D. K. Fowler, S. Wu et al., "Direct Anandamide Activation of TRPV1 Produces Divergent Calcium and Current Responses," Frontiers in Molecular Neuroscience, vol. 10, 2017.

[25] S. Wang, S. Wang, J. Asgar et al., "Ca ," The Journal of Biological Chemistry, vol. 292, no. 20, pp. 8291-8303, 2017.

[26] T. Christoph, G. Bahrenberg, J. De Vry et al., "Investigation of TRPV1 loss-of-function phenotypes in transgenic shRNA expressing and knockout mice," Molecular and Cellular Neuroscience, vol. 37, no. 3, pp. 579-589, 2008.

[27] H. S. Bhatia, N. Roelofs, E. Muñoz, and B. L. Fiebich, "Alleviation of Microglial Activation Induced by p38 MAPK/MK2/PGE2 Axis by Capsaicin: Potential Involvement of other than TRPV1 Mechanism/s," Scientific Reports, vol. 7, no. 1, 2017.

[28] Y.-W. Wu, Y.-P. Bi, X.-X. Kou et al., "17- $\beta$-Estradiol enhanced allodynia of inflammatory temporomandibular joint through upregulation of hippocampal TRPV1 in ovariectomized rats," The Journal of Neuroscience, vol. 30, no. 26, pp. 8710-8719, 2010.
[29] X. Xiao, X.-T. Zhao, L.-C. Xu et al., "Shp-1 dephosphorylates TRPV1 in dorsal root ganglion neurons and alleviates CFAinduced inflammatory pain in rats," Pain, vol. 156, no. 4, pp. 597-608, 2015.

[30] X. Zhang, J. Huang, and P. A. McNaughton, "NGF rapidly increases membrane expression of TRPV1 heat-gated ion channels," EMBO Journal, vol. 24, no. 24, pp. 4211-4223, 2005.

[31] I. Borbiro, D. Badheka, and T. Rohacs, "Activation of TRPV1 channels inhibits mechanosensitive piezo channel activity by depleting membrane phosphoinositides," Science Signaling, vol. 8, no. 363, article no. ra15, 2015.

[32] S. Hong, T. Nguyen, S. Ma, H. Kim, S. Lee, and C. Jang, "TRPV1 modulates morphine-induced conditioned place preference via p38 MAPK in the nucleus accumbens," Behavioural Brain Research, vol. 334, pp. 26-33, 2017.

[33] Y. Liu, Y. Liu, H. Jin et al., "Cold stress-induced brain injury regulates TRPV1 channels and the PI3K/AKT signaling pathway," Brain Research, vol. 1670, pp. 201-207, 2017.

[34] C. A. Ufret-Vincenty, R. M. Klein, M. D. Collins, M. G. Rosasco, G. Q. Martinez, and S. E. Gordon, "Mechanism for phosphoinositide selectivity and activation of TRPV1 ion channels," The Journal of General Physiology, vol. 145, no. 5, pp. 431-442, 2015.

[35] S. Amadesi, G. S. Cottrell, L. Divino et al., "Protease-activated receptor 2 sensitizes TRPV1 by protein kinase Cepsilon- and A-dependent mechanisms in rats and mice," The Journal of Physiology, vol. 575, no. 2, pp. 555-571, 2006.

[36] S. Shan, M.-Y. Qi-Liang, C. Hong et al., "Is functional state of spinal microglia involved in the anti-allodynic and antihyperalgesic effects of electroacupuncture in rat model of monoarthritis?” Neurobiology of Disease, vol. 26, no. 3, pp. 558568, 2007.

[37] S.-Y. Wu, W.-H. Chen, C.-L. Hsieh, and Y.-W. Lin, "Abundant expression and functional participation of TRPV1 at Zusanli acupoint (ST36) in mice: mechanosensitive TRPV1 as an 'acupuncture-responding channel,' BMC Complementary and Alternative Medicine, vol. 14, article 96, 2014.

[38] Z. Zhang, C. Wang, G. Gu et al., "The effects of electroacupuncture at the ST36 (Zusanli) acupoint on cancer pain and transient receptor potential vanilloid subfamily 1 expression in walker 256 tumor-bearing rats," Anesthesia \& Analgesia, vol. 114, no. 4, pp. 879-885, 2012.

[39] W.-H. Chen, J. T. C. Tzen, C. L. Hsieh et al., "Attenuation of TRPV1 and TRPV4 expression and function in mouse inflammatory pain models using electroacupuncture," Evidence-Based Complementary and Alternative Medicine, vol. 2012, Article ID 636848, 2012.

[40] G. R. Ross, A. R. Gade, W. L. Dewey, and H. I. Akbarali, "Opioidinduced hypernociception is associated with hyperexcitability and altered tetrodotoxin-resistant $\mathrm{Na}+$ channel function of dorsal root ganglia," American Journal of Physiology-Cell Physiology, vol. 302, no. 8, pp. C1152-C1161, 2012. 

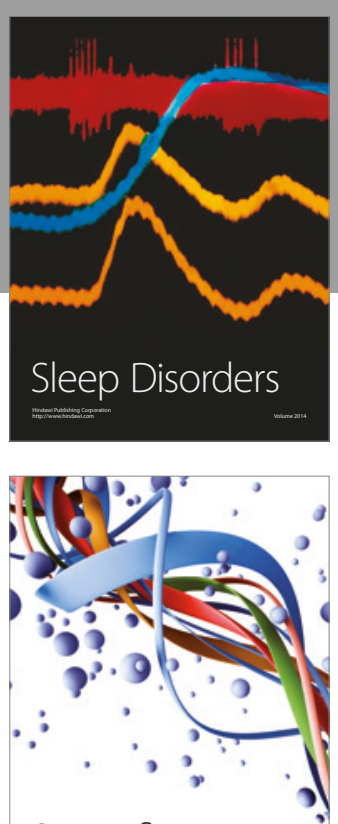

Scientifica
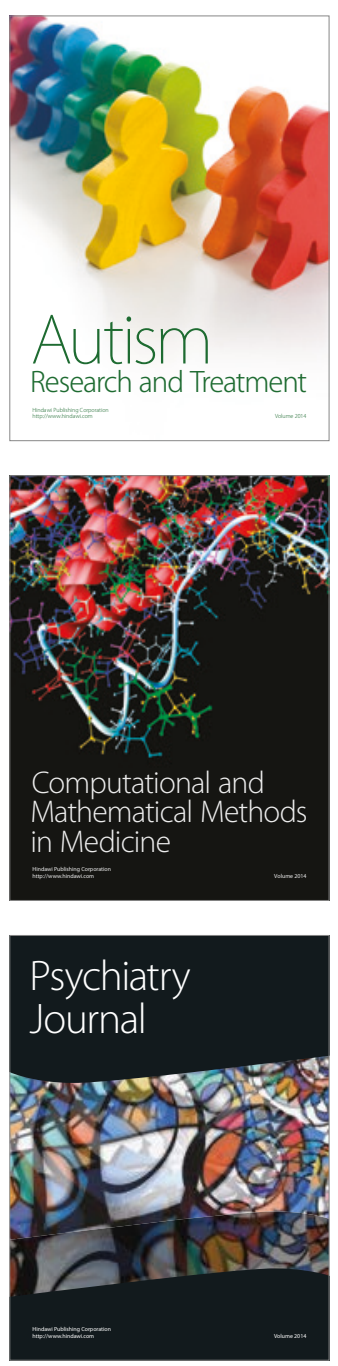
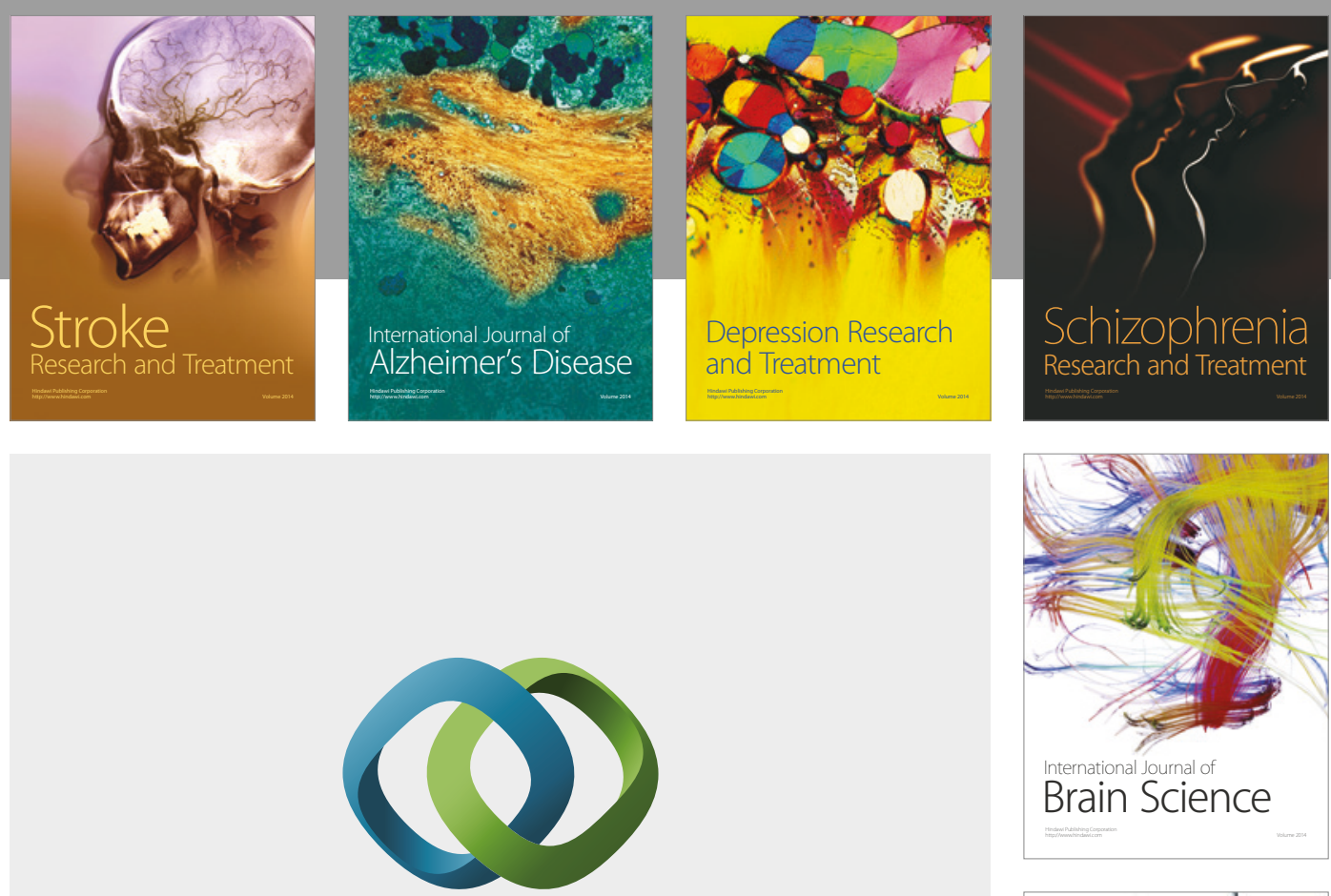

\section{Hindawi}

Submit your manuscripts at

https://www.hindawi.com
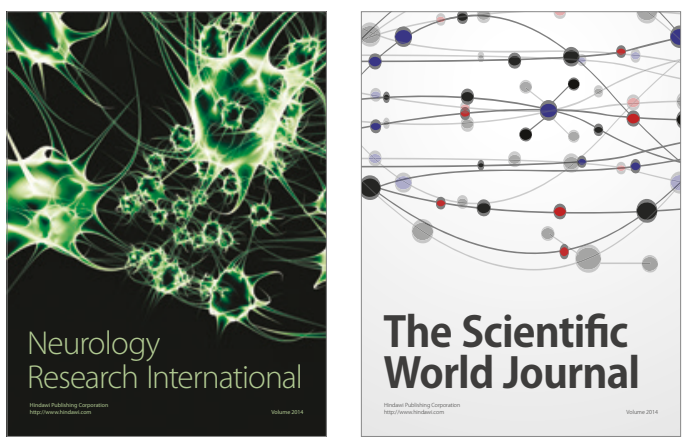

The Scientific World Journal

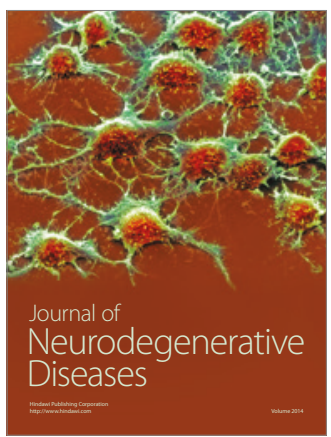

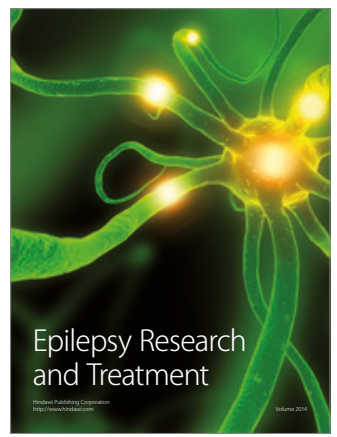

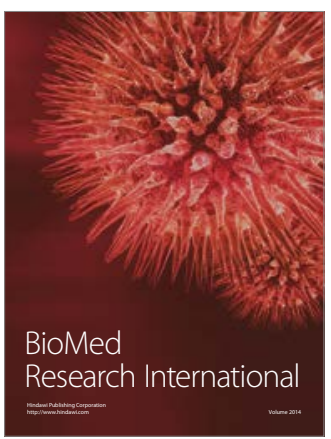

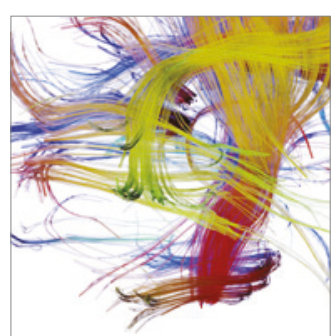

Brain Science

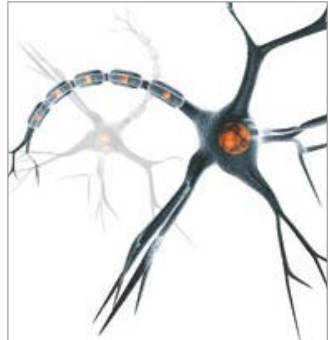

Neural Plasticity
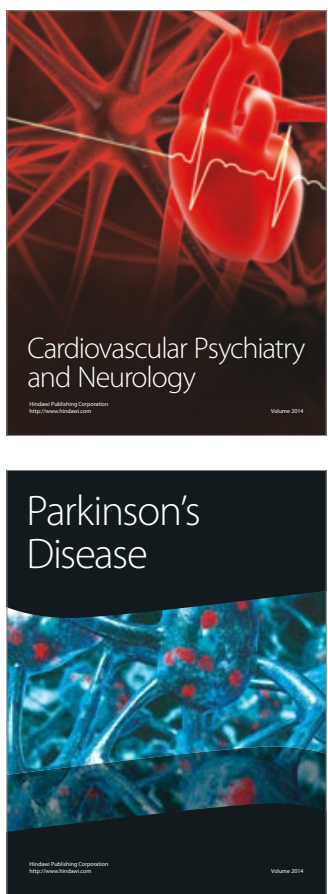